\title{
Influence of Tensor Interaction on Evolution of Nuclear Shells
}

\author{
Rupayan Bhattacharya \\ Department of Physics, University of Calcutta, Kolkata, India \\ Email: rupayanbhattacharya1951@gmail.com
}

Received 2013

\begin{abstract}
Using Skyrme's density dependent interaction the evolution of nuclear shells has been studied in Hartree-Fock formalism. Optimization of the strength of tensor interaction has been done in reproducing the observed splitting of shell model states of ${ }^{40,48} \mathrm{Ca},{ }^{56} \mathrm{Ni}$ and ${ }^{208} \mathrm{~Pb}$. Spin-orbit splitting in Ca-isotopes, ${ }^{56} \mathrm{Ni},{ }^{90} \mathrm{Zr}, N=82$ isotones, Sn-isotopes and evolution of gaps in $Z, N=8,20$ have been reanalyzed with the inclusion of tensor interaction. For doubly shell closed nuclei, it has been observed that tensor interaction is sensitive to spin saturation of nuclear shells.
\end{abstract}

Keywords: Tensor Interaction; Hartree-Fock Theory; Nuclear Shells

\section{Introduction}

The important role of the tensor component of the nucleon-nucleon interaction information of shells beyond the conventional ones was pointed out in the seventies [1-6]. Study of shell evolution and modification of magic numbers in exotic nuclei after inclusion of the tensor component of the nucleon-nucleon interaction in the selfconsistent mean field calculations generated lot of interest in the nuclear structure study circle.

In order to study the evolution of shells with the inclusion of tensor force in Skyrme-Hartree-Fock theory we have studied the spin-orbit splitting of some shell model states of doubly shell closed nuclei, shell gap evolutions of $Z, N=8,20$ nuclei, difference in single particle(-hole) energy levels near the Fermi surfaces of $Z=50$ isotopes, $\mathrm{Ca}$ - isotopes and $N=82$ isotones.

\section{Theoretical Formulation}

The tensor interaction in HF theory is given by [5]

$$
\begin{aligned}
& V_{\mathrm{T}}=\frac{\tau}{2}\left\{\begin{array}{c}
{\left[\left(\sigma_{1}, k^{\prime}\right)\left(\sigma_{2^{*}} k^{\prime}\right)-\frac{1}{8}\left(\sigma_{1^{2}}, \sigma_{2}\right) k^{\prime 2}\right] \tilde{\delta}\left(r_{1}-r_{2}\right)+} \\
\delta\left(r_{1}-r_{2}\right)\left[\left(\sigma_{1}, k\right)\left(\sigma_{2}, k\right)-\frac{1}{8}\left(\sigma_{1}, \sigma_{2}\right) k^{2}\right]
\end{array}\right\} \\
& +U\left(\sigma_{1} \cdot k^{\xi}\right) \sigma\left(r_{1}-r_{2}\right)\left(\sigma_{1} \cdot k\right)-\frac{1}{g}\left(\sigma_{1}, \sigma_{2}\right) \\
& \left.\mathbf{X}\left[\boldsymbol{k}^{y} \cdot \delta\left(x_{1}-r_{2}\right) \boldsymbol{k}\right]\right\}
\end{aligned}
$$

The expressions for proton (- neutron) spin-orbit potential is

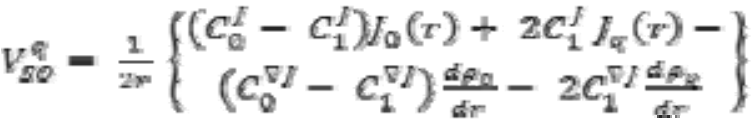

$$
\begin{aligned}
& \text { L. S } \\
& C_{0}^{\square /}=-\frac{3}{4} W_{0}
\end{aligned}
$$

The final spin-orbit potential component is given by

$$
V_{s . Q}^{q}=\frac{W_{Q}}{2 r}\left(2 \frac{d \rho_{q}}{d r}+\frac{d \rho_{q}}{d r}\right)+\left(\alpha \frac{l_{q}}{r}+\beta \frac{I_{q}}{r}\right)
$$

where $J_{q\left(q^{\prime}\right)}(r)$ is the proton or neutron spin-orbit density defined as

$$
\begin{aligned}
& l_{Q}\left(q^{r}\right)(r)=\frac{1}{4 \pi r^{3}} \sum_{k} v_{i}^{2}(2 j+1) \\
& {\left[h_{i}\left(l_{i}+1\right)-L_{i}\left(l_{i}+1\right)-\frac{3}{4}\right] R_{i}^{2}(r)}
\end{aligned}
$$

We have used different sets of parameters (SKP [8], SLY5 [9], SKX [10], KDEO [11]) for our calculations. A schematic pairing force of BCS type has been used to take care of pairing correlation. To get the optimized spin-orbit constant $c_{0}$, variational studies of the spin-orbit splitting of $1 f_{7 / 2}-1 f_{5 / 2}, 2 p_{3 / 2}-2 p_{1 / 2}$ and $1 d_{5 / 2}-$ $1 \mathrm{~d}_{3 / 2}$ levels were performed for isoscalar spin-saturated $N=Z$ nucleus ${ }^{40} \mathrm{Ca}$. It has been observed that the separation energies of the spin-orbit doublets decrease with reduction in values of $W_{0}\left(-4 / 3 C_{0}\right)$ and a reduction is required for agreement with the experimental results. After fixing the first coupling constant $C_{Q}$, other tensor 
coupling constants were fixed by optimizing the $1 \mathrm{f}_{7 / 2^{-}}$ $1 \mathrm{f}_{5 / 2}$ difference in ${ }^{56} \mathrm{Ni}$ and ${ }^{48} \mathrm{Ca}$ nuclei. Our final adjustment of the tensor coupling constants was done after calculating the spin-orbit splitting of several shell model states near the Fermi-surface of the stable shell closed nuclei ${ }^{208} \mathrm{~Pb}$, a very well studied nucleus experimentally.

\section{Results}

The difference in energies for some spin-orbit partner shell model states near the Fermi-surface of ${ }^{208} \mathrm{~Pb}$ are shown in Table 1 for a few standard Skyrme force parameters from where we see that after inclusion of tensor interaction in SKP set, a good agreement with empirical values has been achieved. In Table 2 we present the calculated splitting of the spin-orbit partners of proton and neutron single particle states ${ }^{16} \mathrm{O},{ }^{40,48} \mathrm{Ca},{ }^{56} \mathrm{Ni},{ }^{90} \mathrm{Zr},{ }^{132} \mathrm{Sn}$ along with the empirical values [12,13]. Neutron single-particle energy differences between $1 i_{13 / 2}$ and $1 h_{9 / 2}$ states in $N=82$ isotones calculated with and without inclusion of tensor force along with experimental values [12] have been presented in Figure 1 In Figure 2 we present the gap evolution for Oxygen $(Z=8)$ isotopes.

Table 1. Splitting of spin-orbit doublets in ${ }^{208} \mathbf{P b}$.

\begin{tabular}{cccccccc}
\hline \multirow{2}{*}{$\mathrm{P}$} & \multicolumn{7}{c}{ Energy in MeV } \\
\cline { 2 - 8 } & Sly5 & Sly4 & KDE & KDEt & SKP & SKPt & EXP \\
\hline$\Delta 1 \mathrm{~h}$ & 5.90 & 6.22 & 5.77 & 4.87 & 5.24 & 5.30 & 5.56 \\
$\Delta 2 \mathrm{~d}$ & 1.98 & 1.89 & 1.99 & 1.57 & 1.55 & 1.41 & 1.33 \\
$\Delta 2 \mathrm{f}$ & 2.68 & 2.61 & 2.70 & 2.17 & 2.15 & 1.98 & 1.93 \\
$\Delta 3 \mathrm{p}$ & 1.05 & 1.02 & 1.04 & 0.80 & 0.76 & 0.69 & 0.84 \\
$\mathrm{~N}$ & & & & & & & \\
$\Delta 1 \mathrm{~h}$ & 5.80 & 5.59 & 5.75 & 5.48 & 5.18 & 5.09 & 5.10 \\
$\Delta 1 \mathrm{i}$ & 7.64 & 7.25 & 7.67 & 7.10 & 6.62 & 6.39 & 6.46 \\
$\Delta 2 \mathrm{f}$ & 3.10 & 1.96 & 3.16 & 2.47 & 2.30 & 2.12 & 2.03 \\
$\Delta 2 \mathrm{~g}$ & 3.71 & 3.57 & 3.77 & 2.96 & 2.93 & 2.73 & 2.51 \\
$\Delta 3 \mathrm{p}$ & 1.22 & 1.13 & 1.25 & 0.94 & 0.83 & 0.74 & 0.90 \\
$\Delta 3 \mathrm{~d}$ & 1.75 & 1.67 & 1.60 & 1.31 & 1.27 & 1.15 & 0.97 \\
\hline
\end{tabular}

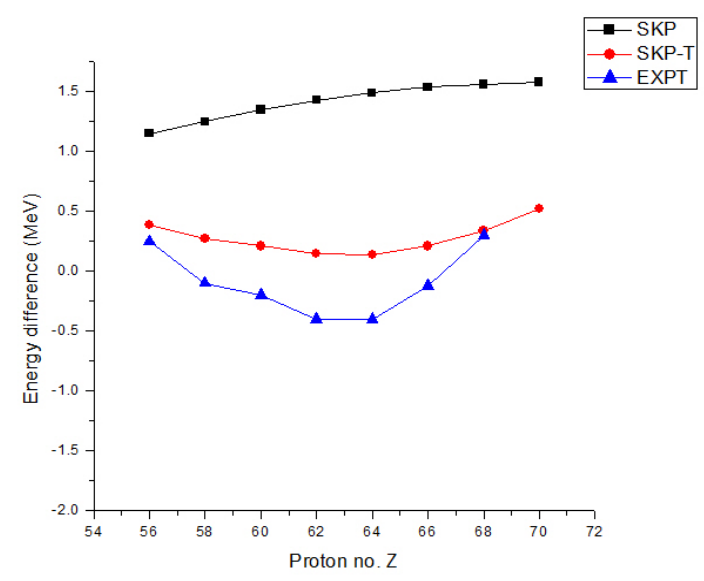

Figure 1. Energy differences of $1 i_{13 / 2}$ and $1 h_{9 / 2}$ states in $N=$ 82 isotones.
Table 2. Calculated values of spin-orbit splitting along with empirical values of doubly shell closed nuclei $\mathrm{O}, \mathrm{Ca}, \mathrm{Sn}, \mathrm{Zr}$.

\begin{tabular}{|c|c|c|c|c|}
\hline \multirow{2}{*}{ Nucleus } & \multirow{2}{*}{ Orbitals } & \multicolumn{2}{|c|}{ Energy difference(T) } & \multirow{2}{*}{$\begin{array}{c}\text { Energy } \\
\text { difference(E) }\end{array}$} \\
\hline & & SKP-T & KDE-T & \\
\hline \multirow{2}{*}{${ }^{16} \mathrm{O}$} & $v \Delta 1 \mathrm{~d}$ & 4.89 & 4.77 & 5.08 \\
\hline & $\pi \Delta 1 \mathrm{~d}$ & 4.65 & 4.54 & 4.97 \\
\hline \multirow{4}{*}{${ }^{40} \mathrm{Ca}$} & $v \Delta 2 p$ & 1.87 & 1.63 & 2.00 \\
\hline & $v \Delta 1 \mathrm{f}$ & 6.45 & 6.24 & 5.64 \\
\hline & $\pi \Delta 2 p$ & 1.65 & 1.46 & 1.72 \\
\hline & $\pi \Delta 1 \mathrm{f}$ & 6.13 & 6.0 & 6.05 \\
\hline \multirow{3}{*}{${ }^{48} \mathrm{Ca}$} & $v \Delta 2 p$ & 2.33 & 2.03 & 1.77 \\
\hline & $v \Delta 1 \mathrm{f}$ & 7.59 & 7.73 & 8.01 \\
\hline & $\pi \Delta 2 p$ & 1.24 & 1.29 & 2.14 \\
\hline \multirow{6}{*}{${ }^{56} \mathrm{Ni}$} & $\pi \Delta 1 \mathrm{f}$ & 5.03 & 5.27 & 4.92 \\
\hline & $v \Delta 2 p$ & 1.83 & 1.69 & 1.88 \\
\hline & $v \Delta 1 \mathrm{f}$ & 6.42 & 6.97 & 6.82 \\
\hline & $\pi \Delta 2 p$ & 1.58 & 1.52 & 1.83 \\
\hline & $v \Delta 1 \mathrm{f}$ & 6.09 & 6.55 & 7.01 \\
\hline & $v \Delta 2 d$ & 2.65 & 2.53 & 2.43 \\
\hline \multirow{3}{*}{${ }^{90} \mathrm{Zr}$} & $v \Delta 1 \mathrm{~g}$ & 7.34 & 7.55 & 7.07 \\
\hline & $\pi \Delta 2 \mathrm{~d}$ & 1.69 & 1.72 & 2.03 \\
\hline & $\pi \Delta 1 \mathrm{~g}$ & 5.28 & 5.36 & 5.56 \\
\hline \multirow{7}{*}{${ }^{132} \mathrm{Sn}$} & $\pi \Delta 1 \mathrm{f}$ & 3.84 & 3.94 & 4.56 \\
\hline & $v \Delta 2 \mathrm{f}$ & 2.68 & 2.45 & 2.00 \\
\hline & $v \Delta 3 p$ & 0.93 & 0.75 & 0.81 \\
\hline & $v \Delta \mathrm{h}$ & 7.37 & 7.44 & 6.68 \\
\hline & $v \Delta 2 \mathrm{~d}$ & 2.08 & 2.09 & 1.93 \\
\hline & $\pi \Delta 2 \mathrm{~d}$ & 1.72 & 1.86 & 1.75 \\
\hline & $\pi \Delta 1 \mathrm{~g}$ & 4.62 & 5.15 & 5.33 \\
\hline
\end{tabular}

Empirical values are taken from ref. [12] and [13]

In Figure 2 we have presented the gap evolution for $Z$ $=8$ with and without tensor forces. For comparison experimental data sets have also been shown. The gap here means the difference in single particle energies of the first particle state above and the last hole state below the Fermi surface of the nuclei under consideration. Since we are dealing with shell closed nuclei, the shell model nomenclature has been used for the particle (-hole) states. For the $Z=8$ isotopes and $N=8$ (Figrue 3) isotones the energy difference between the first unoccupied proton (neutron) level $1 d_{5 / 2}$ and the last occupied state $1 p_{1 / 2}$ constitute the gap. Inclusion of tensor interaction changes the curvature of the shell evolution of the graph correctly and also produces the kink at $N=16$ indicating a shell closure at ${ }^{24} \mathrm{O}$ as observed in the recent experimental results. 
The gap for $Z=20$ isotopes and $N=20$ isotonic chain is the energy difference between the proton (neutron) single particle states $1 f_{7 / 2}$ and $1 d_{3 / 2}$. In the gap evolution of $Z=20$ isotopes as shown in Figure 4, inclusion of tensor interaction increases the gap, as more and more neutrons are added in the system. We find the gap reaches a maximum at $N=32$. Dinca et al. [14] have studied the even ${ }^{52-56} \mathrm{Ti}$ isotopes with intermediate-energy Coulomb excitation and absolute $B\left(E 2 ; 0^{+} \rightarrow 2_{1}{ }^{+}\right)$transition rates have been obtained. These data confirm the presence of a sub-shell closure at neutron number $N=32$ in neutron-rich nuclei above the doubly magic nucleus ${ }^{48} \mathrm{Ca}$.

In Figure 5 we present the gap evolution of $N=20$ isotones for $\mathrm{Si}$ to $\mathrm{Fe}$ nuclei. Though in the experimental data one observes a peak at $Z=20$, we have obtained a change in the gradient at that point when tensor interaction is taken into consideration

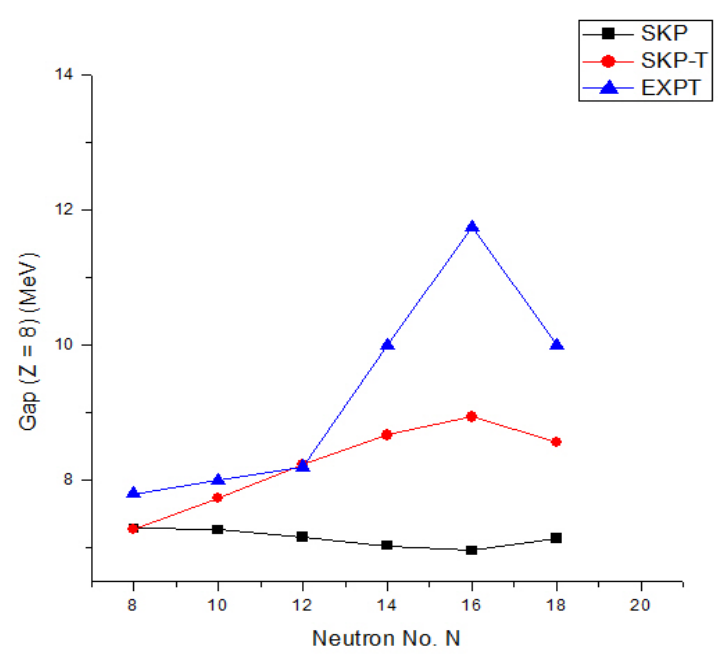

Figure 2. Comparison of calculated gap evolution of $Z=8$ isotones with and without tensor interaction and experimental data.

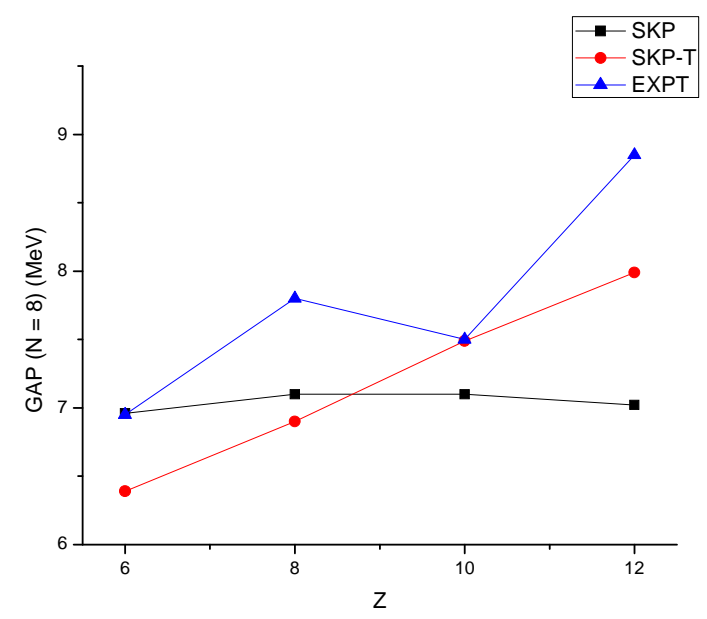

Figure 3. Gap evolution of $N=8$ isotones with and without tensor interaction along with experimental data.

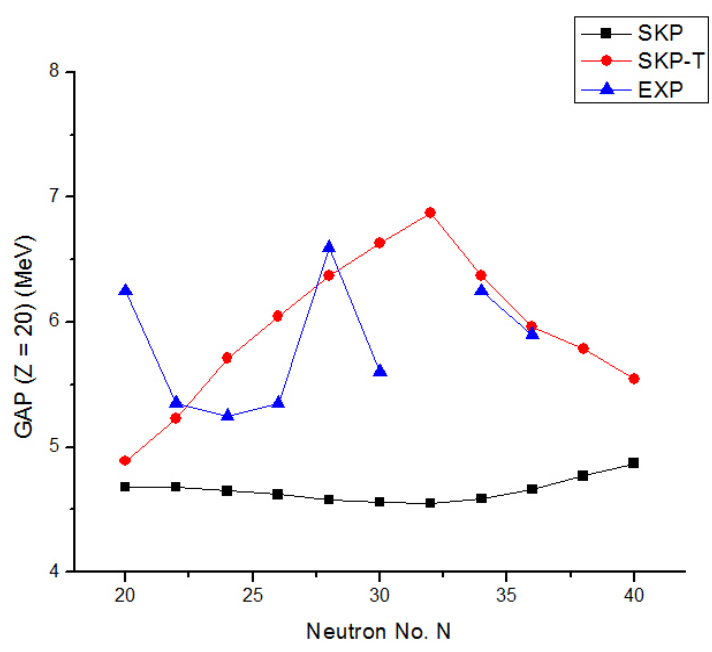

Figure 4. Comparison of calculated gap evolution of $Z=20$ isotones with and without tensor interaction and experimental data.

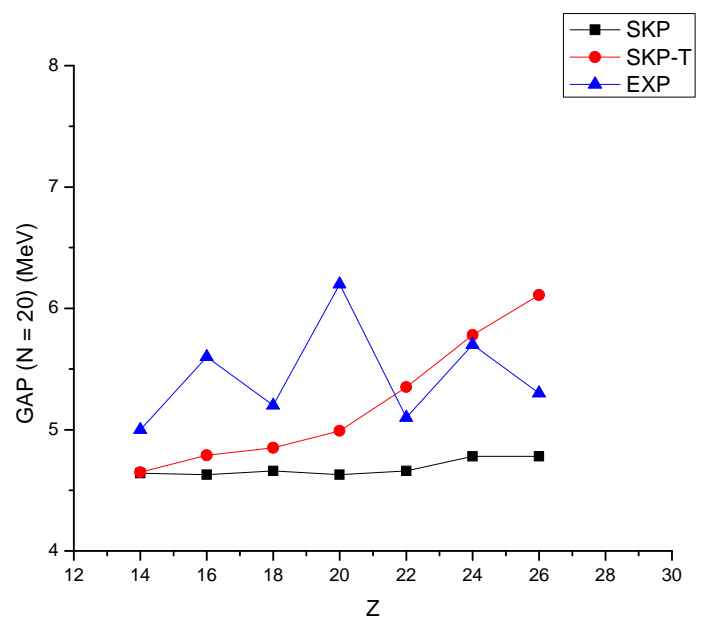

Figure 5. Gap evolution of $N=20$ isotones with and without tensor interaction and experimental data.

\section{Conclusions}

It has been shown in this work that inclusion of tensor interaction in the Skyrme-Hartree-Fock theory induces a change in the spin-orbit potential of nucleus and hence improves the single particle structure of shell closed nucleus considerably and the optimized coefficients of tensor interaction reproduces the evolution of shell gaps for $Z, N=8,20$, two most important low mass shell closed nuclei and generates the spin-orbit splitting of some shell model states near the Fermi surface of some shell closed nuclei. Furthermore, it also reproduces the trend in change of energy differences in $1 h_{11 / 2}$ and $1 g_{9 / 2}$ states of $\mathrm{Sn}$ isotopes.

\section{Acknowledgements}

The author thanks the UGC for support by the Emeritus 
Fellowship [No. F.6-34/2011(SA - II)].

\section{REFERENCES}

[1] D. Vautherin and D. M. Brink, "Hartree-Fock Calculations with Skyrme's Interaction," Physics Letters B, Vol. 32, No. 3, 1970, pp. 149-153. doi:10.1016/0370-2693(70)90458-2

[2] D. Vautherin and D. M. Brink, "Hartree-Fock Calculations with Skyrme's Interaction. I . Spherical Nuclei," Physical Review C, Vol. 5, No. 3, 1972, pp. 626-647. doi:10.1103/PhysRevC.5.626

[3] T. Otsuka, et al., "Magic Numbers in Exotic Nuclei and Spin-Isospin Properties of the NN Interaction," Physical Review Letters, Vol. 87, No. 8, 2001, 082502. doi:10.1103/PhysRevLett.87.082502

[4] G. G. Colo, H. Sagawa, S. Fracasso and P. F. Bortignon, "Spin-Orbit Splitting and the The Tensor Component of the Skyrme Interaction," Physics Letters B, Vol. 646, No. 5-6, 2007, pp. 227-231. doi.org/10.1016/i.physletb.2007.01.033

[5] D. M. Brink and F. Stancu, "Evolution of Nuclear Shells with the Skyrme Density Dependent Interaction," Physical Review C, Vol. 75, 2007, 064311. doi:10.1103/PhysRevC.75.064311

[6] T. Lesinski, M. Bender, K. Bennaceur, T. Duguet and J. Meyer, "Tensor Part of The Skyrme Energy Density Functional: Spherical Nuclei," Physical Review C, Vol. 76, 2007, p. 014312. doi:10.1103/PhysRevC.76.014312

[7] M. Zalewski, J. Dobaczewski, W. Satula and T. R. Werner, "Spin-Orbit and Tensor Mean-Field Effects on
Spin-Orbit Splitting Including Self-Consistent Core Polarization," Physical Review C, Vol. 77, 2008, p. 024316.doi:10.1103/PhysRevC.77.024316

[8] Y. Z. Wang, J. Z. Gu, J. M. Dong and X. Z. Zhang, "Systematic Study of Tensor Effects in Shell Evolution," Physical Review C, Vol. 83, 2011,054305. doi:10.1103/PhysRevC.83.054305

[9] J. Dobaczewski, H. Flocard and J. Treiner, "Hartree-Fock-Bogolyubov Description of Nuclei Near The Neutron-Drip Line," Nuclear Physics A, Vol. 422, No. 1, 1984, pp. 103-139. doi:10.1016/0375-9474(84)90433-0

[10] E. Chabanat, P. Bonche, P. Haensel, J. Meyer and R. Schaeffer, "A Skyrme Parametrization from Subnuclear to Neutron Star Densities Part II. Nuclei Far from Stabilities," Nuclear Physics A, Vol. 635, No. 1-2, 1998, pp. 231-256. doi:10.1016/S0375-9474(98)00180-8

[11] B. A. Brown, "New Skyrme Interaction for Normal and Exotic Nuclei," Physical Review C, Vol. 58, 1998, pp. 220-231. doi:10.1103/PhysRevC.58.220

[12] B. K. Agrawal, S. Shlomo and V. Kim Au, "Determination of the Parameters of A Skyrme Type Effective Interaction Using the Simulated Annealing Approach," Physical Review C, Vol. 72, 2005, 014310 doi:10.1103/PhysRevC.72.014310

[13] N. Schwierz, I. Wiedenhover and A. Volya, arXiv:0709.3525

[14] A. Oros, Ph.D thesis, University of Koln, 1996.

[15] D. C. Dinca, et al., "Reduced Transition Probabilities to the First 2 State in ${ }^{52,54,56} \mathrm{Ti}$ and Development of Shell Closures at $\mathrm{N}=32$, 34," Physical Review C, Vol. 71, 2005, 041302.doi:10.1103/PhysRevC.71.041302 